\title{
Clinics in diagnostic imaging (179)
}

Shi Xian Shawn Kok ${ }^{1}$, MBBS, FRCR, Tien Jin $\underline{T a n}^{1}$, MBBS, FRCR
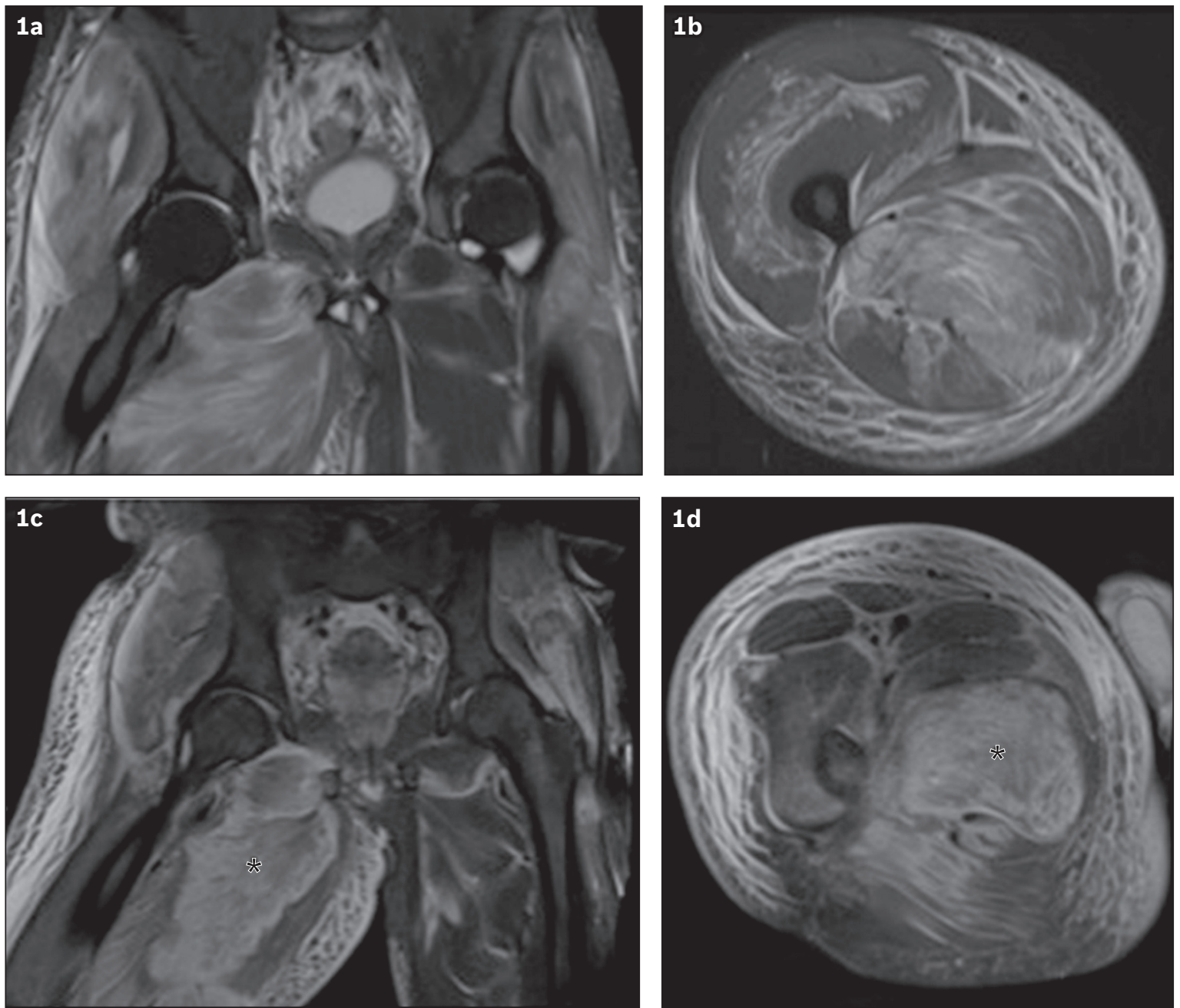

Fig. 1 Coronal and axial turbo inversion recovery magnitude MR imaging sequences of the pelvis and right thigh ( $a$ \& $b$ ) at the time of admission and (c \& d) 11 days following admission when the patient's symptoms worsened.

\section{CASE PRESENTATION}

A 32-year-old Chinese man with a history of recurrent seizures, depression and multiple suicide attempts by drug overdose presented to the emergency department with severe right lower limb pain and swelling of three days' duration. He suffered an episode of seizure upon admission. On examination, the right thigh and calf were significantly swollen and tender. There was no significant neurovascular compromise of the right lower limb. Laboratory markers revealed significant elevation of serum creatine kinase (CK) level at $>4,100 \mathrm{U} / \mathrm{L}$ and urine myoglobinuria at 1,409 $\mu \mathrm{g} / \mathrm{L}$. Serum creatinine was also elevated at $329 \mu \mathrm{mo} / \mathrm{L}$. Mild hyperkalaemia was noted at $5.5 \mathrm{mmol} / \mathrm{L}$. A review of the patient's medications revealed that he had been on multiple prescription drugs, notably the selective serotonin receptor inhibitor (SSRI) escitalopram $15 \mathrm{mg}$ every night for depression. Magnetic resonance (MR) imaging of the pelvis and right thigh was performed at the time of admission (Figs. 1a \& b), as well as 11 days later, when the patient's symptoms worsened (Figs. 1c \& d). What do the images show? What is the diagnosis? 


\section{IMAGE INTERPRETATION}

At the time of admission, coronal and axial turbo inversion recovery magnitude (TIRM) sequences show diffuse hyperintensity and swelling in the bilateral gluteal and right adductor compartments (Fig. 1a) and, to a lesser extent, the quadriceps and hamstring muscles (Fig. 1b), in keeping with extensive myositis. The general architecture of the muscle remains fairly preserved. Coronal and axial TIRM sequences 11 days following admission show more confluent and discrete foci of heterogeneous hyperintensity in the right adductor compartment, with central areas of intermediate signal intensity (black asterisks in Figs. 1c \& d), representing interval development of myonecrosis.

\section{DIAGNOSIS}

Severe rhabdomyolysis complicated by myonecrosis.

\section{CLINICAL COURSE}

The patient was aggressively rehydrated intravenously, but his renal function and serum CK levels failed to improve after two days. He was then transferred to the medical intensive care unit and commenced on continuous veno-venous haemofiltration $(\mathrm{CVVH})$, with subsequent improvements in his renal function and serum CK levels. His right lower limb neurovascular status remained intact, which excluded the possibility of an evolving compartment syndrome. No attributable autoimmune or viral cause of the patient's rhabdomyolysis was elucidated. He was scheduled for further evaluation with electromyography and muscle biopsy, but these were not performed, as the patient requested for hospital discharge against medical advice.

\section{DISCUSSION}

Acute rhabdomyolysis should be recognised early and promptly treated, as it has potentially deleterious consequences. The condition occurs as a result of striated muscle breakdown, which causes the release of various intracellular contents, including CK, aldolase, myoglobin, potassium, carbonic anhydrase and lactate dehydrogenase. ${ }^{(1-3)}$ Although the spectrum of clinical presentation is varied, the symptoms of cramp-like pain, swelling and loss of function of the affected muscle group (more commonly the large muscle groups of the thigh, pelvis or paraspinal region) usually predominate. There may be accompanying nonspecific symptoms such as malaise, lethargy and gastrointestinal discomfort. Teacoloured urine is a classical description in textbooks and is due to myoglobinuria. ${ }^{(2,3)}$

Laboratory markers greatly aid in the diagnosis of rhabdomyolysis. A markedly elevated serum CK level, in particular the CK-MM subtype that is found in striated muscle, is highly sensitive for muscle damage. The presence of urine myoglobinuria is an important additional finding for confirmation, although the absence of myoglobinuria does not exclude rhabdomyolysis. Muscle biopsy, while confirmatory, is usually not required. ${ }^{(1-3)}$

The causes of rhabdomyolysis include substance abuse (alcohol and illicit drugs), trauma, excessive muscular activity, prescription drug use (e.g. salicylates, fibrates, statins, anaesthetic and antidepressive agents), seizures and infection. ${ }^{(2-4)}$ In the present case, our patient was known to be on the SSRI escitalopram, for treatment of depression. SSRIs have an established association with rhabdomyolysis.(4) A variety of mechanisms have been postulated to explain drug-induced rhabdomyolysis. Toxins may induce vasospasm, cause involuntary muscle contraction, affect muscular metabolism and interfere with the metabolism of adenosine triphosphate. ${ }^{(4)}$

More importantly, the attending clinician should be aware of the potential complications of rhabdomyolysis, which include electrolyte abnormalities, hypovolaemia, acute renal failure, disseminated intravascular coagulation, metabolic acidosis, compartment syndrome and liver dysfunction. ${ }^{(1-3)}$ In a retrospective evaluation of 475 hospitalised patients with rhabdomyolysis, Melli et al found that acute renal failure secondary to myoglobinuria was present in nearly half of the patients $(46 \%)$. ${ }^{(3)}$ Likewise, our patient also developed acute renal failure, and his renal function improved only after he was treated with CVVH.

MR imaging, computed tomography (CT) and ultrasonography may be helpful in the radiological evaluation of rhabdomyolysis. Muscle abnormalities may be broadly classified into three patterns: muscle oedema, fatty infiltration and mass lesion. ${ }^{(5,6)} \mathrm{MR}$ imaging has superior soft-tissue contrast resolution and the highest sensitivity among the different imaging modalities available for the detection of rhabdomyolysis. ${ }^{(7,8)}$ Diffuse T2-weighted hyperintensity with corresponding $\mathrm{T} 1$-weighted hypointensity in the affected muscle groups, the most common finding described on MR imaging, represents diffuse oedema. Inversion recovery sequences, such as TIRM and short-tau inversion recovery (STIR), are helpful in accentuating contrast between abnormal and normal muscle, as well as adjacent fat, due to inherent fat suppression. Diffuse oedema in rhabdomyolysis may progress to myonecrosis and appear 'mass-like'.(5)

Two distinct types of MR imaging findings may be seen in rhabdomyolysis, as proposed by Lu et al in 2007. ${ }^{(7)}$ In Type 1, which occurs in cases of overexertion, the involved muscles usually appear homogeneously isointense to hyperintense on T1-weighted imaging, and homogeneously hyperintense on T2-weighted and STIR sequences. Type 2 is encountered in cases of direct trauma, vascular occlusion, carbon monoxide poisoning, as well as alcohol and illicit drug abuse. Muscles affected by Type 2 rhabdomyolysis usually present with homogeneous or heterogeneous hyperintense signal on T1-weighted imaging and heterogeneously hyperintense signal on T2-weighted sequences. This is in contrast to Type 1 MR imaging findings, in which the signal change of the affected muscles on both T1- and T2-weighted images is usually homogeneous. A characteristic 'stippled' sign may also be present, causing a dot-like or streaky linear appearance within a region of rim enhancement on contrast-enhanced MR imaging. The appearance of Type 2 findings has been postulated to be due to remnant muscle fibres or inflammatory vasculature within necrotic muscle, representing areas of myonecrosis. (7)

Areas of intramuscular haemorrhage, which appear hyperintense on T1-weighted images and hypointense on 

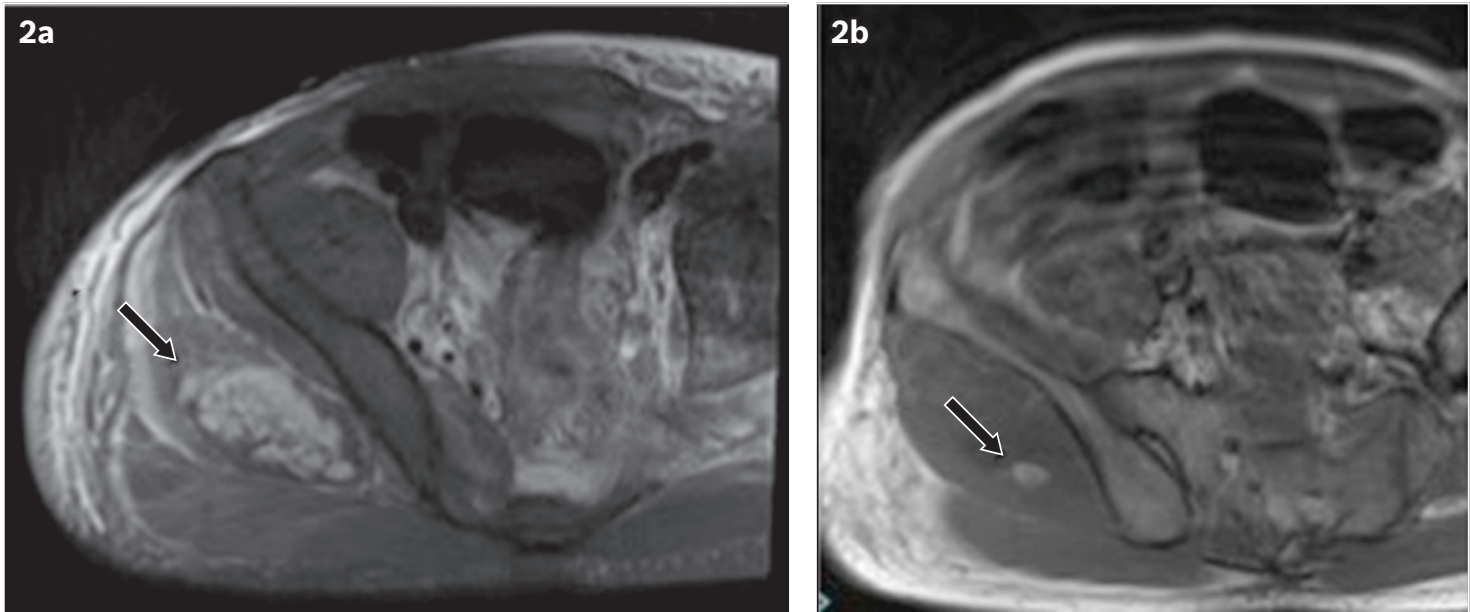

Fig. 2 (a) Axial turbo inversion recovery magnitude MR image shows focal myonecrosis in the gluteus medius (arrow). (b) Axial T1-weighted MR image shows focal hyperintensity within the region of myonecrosis, likely representing haemorrhage (arrow).
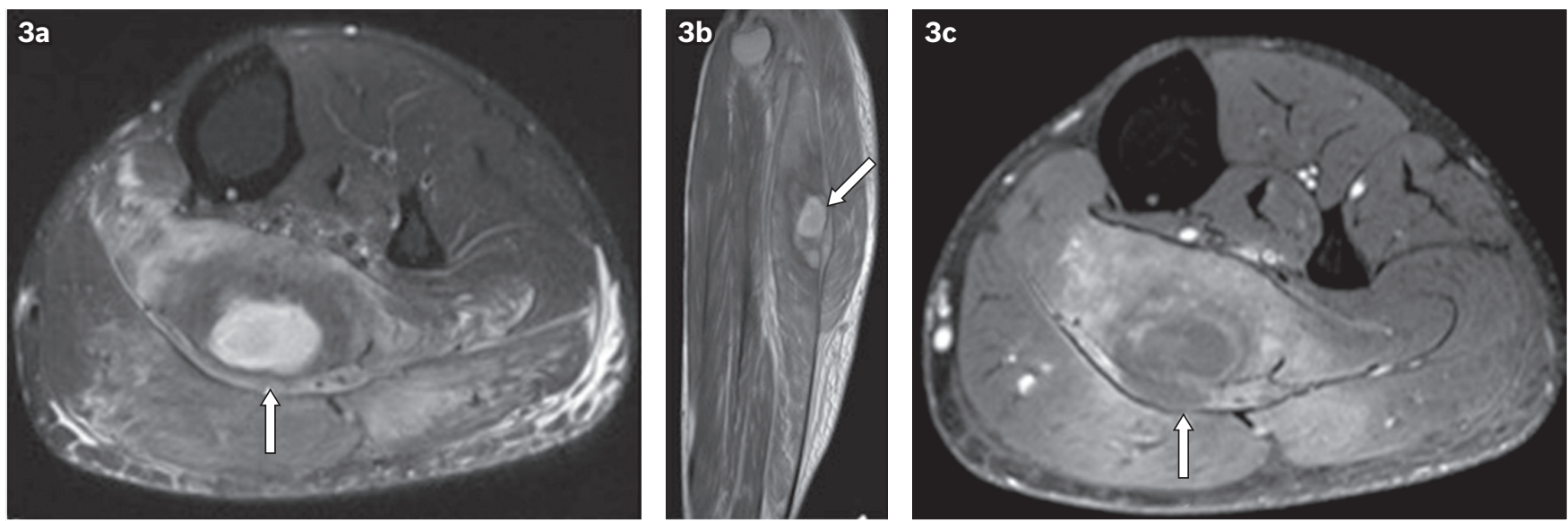

Fig. 3 A 36-year-old Malay man with poorly controlled diabetes mellitus and pyomyositis of the left calf. (a) Axial turbo inversion recovery magnitude and (b) sagittal proton density-weighted MR images show a hyperintense focus (arrows) within the left soleus with surrounding muscle oedema. (c) Axial T1 fat-saturated post-contrast MR image shows a rim-enhancing collection in the soleus (arrow) with enhancement of the surrounding soleus and gastrocnemius.

gradient-recalled echo images, have also been described in cases of rhabdomyolysis. ${ }^{(8,9)}$ Similarly, follow-up MR imaging of our patient showed an area of myonecrosis in the right gluteus medius (Fig. 2a), with focal T1-weighted hyperintensity within, likely representing haemorrhage (Fig. 2b).

On CT imaging, involved muscle groups usually demonstrate low attenuation due to underlying oedema, with occasional rim-enhancement on contrast-enhanced imaging. However, these are nonspecific imaging findings that can also be seen in pyomyositis and infiltrative intramuscular neoplasms. ${ }^{(7,10,11)}$ On ultrasonography, affected muscle groups generally appear hypoechoic, with effacement of the normal striated architecture of the muscle fibres, although these findings are also nonspecific. ${ }^{(11,12)}$

In the absence of relevant clinical history and laboratory markers, radiological findings of rhabdomyolysis may be difficult to distinguish from those of inflammatory myopathy, muscle infection, necrotising fasciitis or other conditions associated with myonecrosis such as diabetes mellitus or sickle cell crisis. ${ }^{(5,6,9,13-18)}$ Nonetheless, certain clinical clues and radiological patterns may help to guide imaging interpretation. For example, inflammatory myopathies secondary to autoimmune causes, such as polymyositis or dermatomyositis, usually present with slowly progressive onset of weakness and classically involve the symmetrical proximal muscles, which appear hyperintense on fluid-sensitive sequences (e.g. STIR) and fat-saturated contrastenhanced T1-weighted sequences. ${ }^{(13)}$

Muscle infection (i.e. pyomyositis) is occasionally seen in immunocompromised patients and usually involves the large muscle groups of the thigh and pelvis. ${ }^{(14,15)}$ It appears on MR imaging as hyperintense focal lesions with significant perilesional oedema, occasionally progressing to abscess formation (Fig. 3). This is most commonly caused by Staphylococcus aureus, although Streptococcal and Clostridium bacterial strains have been implicated in such infections. ${ }^{(13-15)}$ In the case of necrotising fasciitis, the presence of thick (>3 mm) hyperintensity within the deep fascial planes (particularly the intermuscular fascia) involving multiple compartments is a highly suspicious finding. Gas foci within the deep fascia may be seen as signal voids on gradient-recalled echo images. Deep fascia and muscular contrast enhancement is variable on MR imaging, and the absence of contrast enhancement may be associated with necrosis (Fig. 4). ${ }^{(14)}$ 

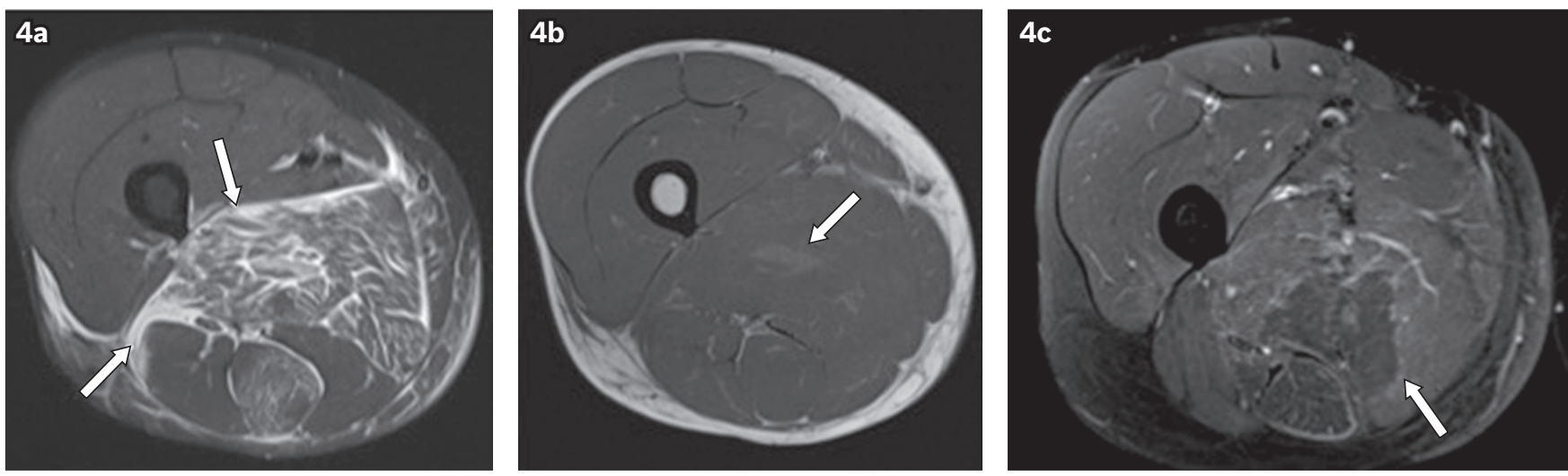

Fig. 4 A 31-year-old intravenous drug user with necrotising fasciitis of the right thigh. (a) Axial turbo inversion recovery magnitude MR image shows a thickened deep fascia and intermuscular fluid in the medial and posterior compartments of the right thigh (arrows). (b) Axial T1-weighted MR image shows mildly raised signal in the adductor magnus, probably due to haemorrhage (arrow). (c) Axial T1 fat-saturated post-contrast MR image shows hypo-enhancing muscle in the medial and posterior compartments of the thigh, suspicious for necrosis (arrow).
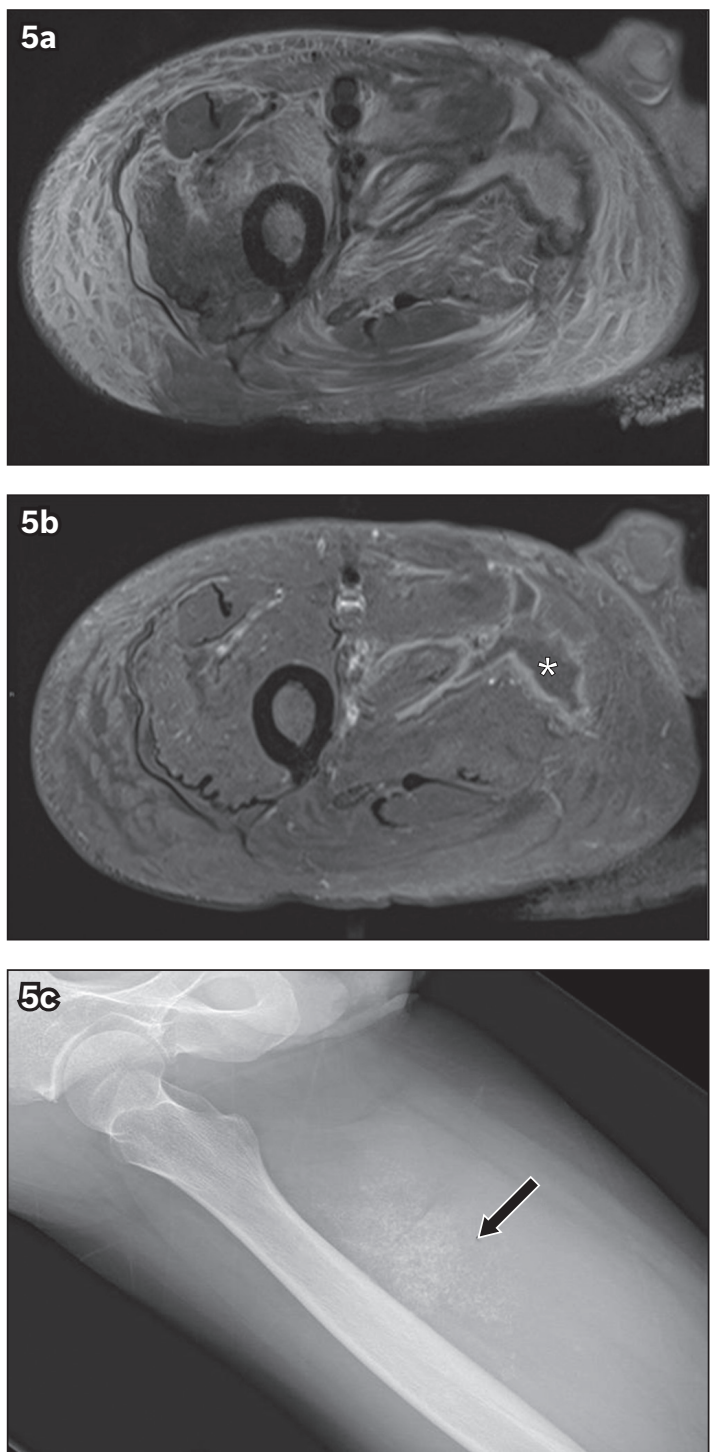

Fig. 5 A 57-year-old Caucasian woman with diabetic myonecrosis of the right thigh. (a) Axial turbo inversion recovery magnitude and (b) axial T1 fat-saturated post-contrast MR images show marked oedema of the anterior and medial compartment muscles, associated with an irregular nonenhancing area of myonecrosis (asterisk in 5b) and subcutaneous oedema. (c) Follow-up radiograph of the thigh shows dystrophic calcifications at the site of myonecrosis (arrow).
Diabetic myonecrosis is an uncommon complication associated with poorly controlled diabetes mellitus, and is thought to result from arteriosclerosis obliterans, causing muscle infarction. ${ }^{(17)}$ The clinical presentation is usually an acute onset of pain, most frequently involving the thigh muscles, without a history of trauma or associated sepsis. ${ }^{(17)} \mathrm{MR}$ imaging findings in acute cases of diabetic myonecrosis include significant muscle oedema and post-contrast enhancement surrounding irregular, and occasionally mass-like, areas of muscle necrosis (Figs. 5a \& b). . $^{(5), 8)}$ Imaging findings of more chronic cases of diabetic myonecrosis include muscular atrophy and dystrophic calcification (Fig. 5c). ${ }^{(18)}$

In summary, this article illustrates the progression of imaging findings in a case of severe rhabdomyolysis that was complicated by myonecrosis. The imaging diagnosis of this condition should be made in tandem with corroborative clinical and laboratory findings, as there is a potential overlap of imaging features with other infective and inflammatory conditions. MR imaging, however, remains clinically useful for assessing the overall extent and severity of rhabdomyolysis, as well as in monitoring for progression or resolution of muscular signal abnormalities.

ABSTRACT A 32-year-old man presented to the emergency department with severe right lower limb pain and swelling of three days' duration. He had multiple prior admissions for recurrent seizures and suicide attempts. Markedly elevated serum creatine kinase levels and urine myoglobinuria were consistent with a diagnosis of rhabdomyolysis. Initial magnetic resonance imaging of the right lower limb revealed diffuse muscle oedema and features of myositis in the gluteal muscles and the adductor, anterior and posterior compartments of the thigh. Follow-up magnetic resonance imaging performed 11 days later showed interval development of areas of myonecrosis and haemorrhage. The causes, clinical presentation and imaging features of rhabdomyolysis are discussed.

Keywords: creatine kinase, myonecrosis, myositis, rhabdomyolysis 


\section{REFERENCES}

1. Vanholder R, Sever MS, Erek E, Lameire N. Rhabdomyolysis. J Am Soc Nephrol 2000; 11:1553-61.

2. Khan FY. Rhabdomyolysis: a review of the literature. Neth J Med 2009; 67:272-83.

3. Melli G, Chaudhry V, Cornblath DR. Rhabdomyolysis: an evaluation of 475 hospitalized patients. Medicine (Baltimore) 2005; 84:377-385.

4. Prendergast BD, George CF. Drug-induced rhabdomyolysis--mechanisms and management. Postgrad Med J 1993; 69:333-6.

5. May DA, Disler DG, Jones EA, Balkissoon AA, Manaster BJ. Abnormal signal intensity in skeletal muscle at MR imaging: patterns, pearls, and pitfalls Radiographics 2000; 20 Spec No: S295-315.

6. Theodorou DJ, Theodorou SJ, Kakitsubata Y. Skeletal muscle disease: pattern of MRI appearances. Br J Radiol 2012; 85:e1298-308.

7. Lu CH, Tsang YM, Yu CW, et al. Rhabdomyolysis: magnetic resonance imaging and computed tomography findings. J Comput Assist Tomogr 2007; 31:368-74

8. Moratalla MB, Braun P, Fornas GM. Importance of MRI in the diagnosis and treatment of rhabdomyolysis. Eur J Radiol 2008; 65:311-5.

9. Cheng YC, Lan HH, Shih CS, Chen CC, Lee SK. Magnetic resonance imaging of rhabdomyolysis: muscle necrosis versus ischemia. J Radiol Sci 2013; 38:143-8

10. Nakahara K, Tanaka H, Masutani K, et al. The value of computed tomography and magnetic resonance imaging to diagnose rhabdomyolysis in acute renal failure. Nephrol Dial Transplant 1999; 14:1564-7.

11. Lamminen AE, Hekali PE, Tiula E, Suramo I, Korhola OA. Acute rhabdomyolysis: evaluation with magnetic resonance imaging compared with computed tomography and ultrasonography. Br J Radiol 1989; 62:326-30.

12. Kaplan GN. Ultrasonic appearance of rhabdomyolysis. AJR Am J Roentgenol 1980; $134: 375-7$.

13. Schulze M, Kötter I, Ernemann U, et al. MRI findings in inflammatory muscle diseases and their noninflammatory mimics. AJR Am J Roentgenol 2009; 192:1708-16.

14. Ali SZ, Srinivasan S, Peh WC. MRI in necrotizing fasciitis of the extremities. $\mathrm{Br}$ J Radiol 2014; 87:20130560.

15. Mitsionis GI, Manoudis GN, Lykissas MG, et al. Pyomyositis in children: early diagnosis and treatment. J Pediatr Surg 2009; 44:2173-8.

16. Pannaraj PS, Hulten KG, Gonzalez BE, Mason EO Jr, Kaplan SL. Infective pyomyositis and myositis in children in the era of community-acquired, methicillin-resistant Staphylococcus aureus infection. Clin Infect Dis 2006; 43:953-60.

17. Horton WB, Taylor JS, Ragland TJ, Subauste AR. Diabetic muscle infarction: a systematic review. BMJ Open Diabetes Res Care 2015; 3:e000082.

18. Chaudhry AA, Baker KS, Gould ES, Gupta R. Necrotizing fasciitis and its mimics: what radiologists need to know. AJR Am J Roentgenol 2015; 204:128-39. 


\section{SINGAPORE MEDICAL COUNCIL CATEGORY 3B CME PROGRAMME} (Code SMJ 201708B)

Question 1. Regarding the diagnosis of acute rhabdomyolysis:

(a) Clinical symptoms usually consist of cramp-like pain, swelling and loss of function.

(b) Laboratory markers such as elevated serum creatine kinase levels and urine myoglobinuria are helpful in diagnosis.

(c) Muscle biopsy is always indicated for definitive diagnosis.

(d) Imaging is helpful in evaluating the anatomical extent and complications of rhabdomyolysis.

Question 2. The following are known clinical complications of rhabdomyolysis:
(a) Acute renal failure.
(b) Disseminated intravascular coagulation.
(c) Diabetes insipidus.
(d) Compartment syndrome.

Question 3. Regarding the radiological evaluation of acute rhabdomyolysis:

(a) Magnetic resonance (MR) imaging has superior soft-tissue resolution in the evaluation of muscle abnormality.

(b) Computed tomography has high specificity in the diagnosis of acute rhabdomyolysis.

(c) Muscle oedema may appear as hypoechoic on ultrasonography.

(d) It is often difficult to distinguish the radiological findings of rhabdomyolysis from those of other infective and inflammatory muscle conditions.

Question 4. The following imaging findings may be seen in MR imaging evaluation of acute rhabdomyolysis:

(a) Homogeneous hyperintense signal on T2-weighted imaging in Type 1 rhabdomyolysis.

(b) Focal areas of raised T1-weighted hyperintensities suggestive of intramuscular haemorrhage.

(c) Raised T1-weighted signal suggestive of muscle oedema.

(d) A 'stippled' appearance in Type 2 rhabdomyolysis.

Question 5. Regarding the clinico-radiological findings of other muscle abnormalities:

(a) Necrotising fasciitis typically spares the deep fascial planes.

(b) Multi-compartmental involvement is typical in necrotising fasciitis.

(c) Diabetes mellitus is associated with myonecrosis.

(d) Inflammatory myopathies (such as polymyositis, dermatomyositis) tend to involve proximal muscle groups.

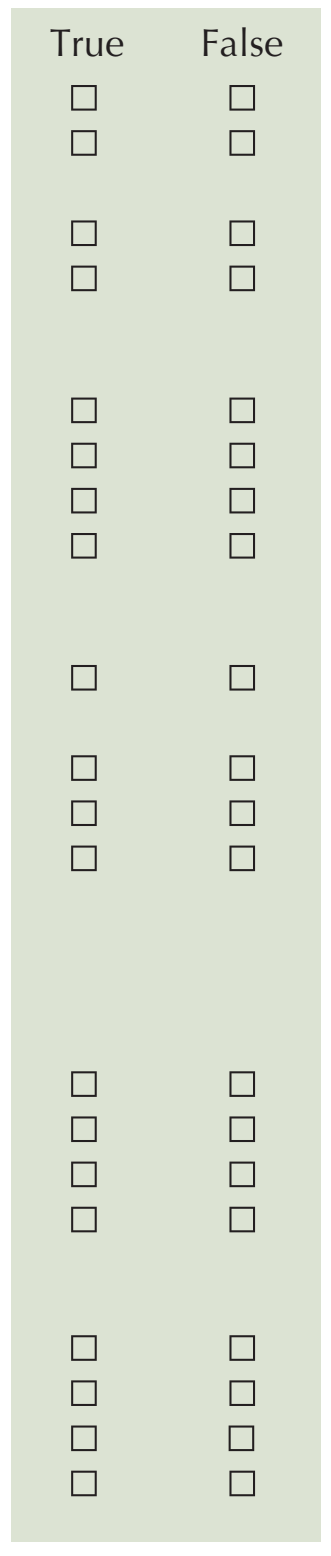

\section{Doctor's particulars:}

Name in full:

MCR no.:

Specialty:

Email:

\section{SUBMISSION INSTRUCTIONS:}

Visit the SMJ website: http://www.smj.org.sg/current-issue and select the appropriate quiz. You will be redirected to the SMA login page.

For SMA member: (1) Log in with your username and password (if you do not know your password, please click on 'Forgot your password?'). (2) Select your answers for each quiz and click 'Submit'.

For non-SMA member: (1) Create an SMJ CME account, or log in with your SMJ CME username and password (for returning users). (2) Make payment of SGD 21.40 (inclusive of $7 \%$ GST) via PayPal to access this month's quizzes. (3) Select your answers for each quiz and click 'Submit'.

RESULTS:

(1) Answers will be published online in the SMJ October 2017 issue. (2) The MCR numbers of successful candidates will be posted online at the SMJ website by 9 October 2017. (3) Passing mark is $60 \%$. No mark will be deducted for incorrect answers. (4) The SMJ editorial office will submit the list of successful candidates to the Singapore Medical Council. (5) One CME point is awarded for successful candidates.

Deadline for submission: (August 2017 SMJ 3B CME programme): 12 noon, 2 October 2017. 